\title{
Trimethoprim Resistance Conferred by W Plasmids in Enterobacteriaceae
}

\author{
By NAOMI DATTA AND R. W. HEDGES \\ Department of Bacteriology, Royal Postgraduate Medical School, \\ Du Cane Road, London, WI2 oHS
}

(Accepted for publication 29 April 1972)

SUMMARY

High-level resistance to trimethoprim (minimum inhibitory concentration $>1000 \mu \mathrm{g} / \mathrm{ml}$ ) was conferred by $\mathrm{R}$ factors of the $\mathrm{W}$ compatibility group in Escherichia coli and Klebsiella spp. isolated from patients in three London hospitals. We suggest that we are observing the early stages in the spread of a new $\mathrm{R}$ factor.

\section{INTRODUCTION}

Trimethoprim, a synthetic inhibitor of folic acid metabolism in bacteria, has been used for the treatment of human infections in Western Europe for 3 to 4 years. The occurrence of trimethoprim-resistant enteric bacteria has been reported (Lebek \& Weidmer, 1971; Fleming, Datta \& Grüneberg, I972; Lacey, Gillespie, Bruten \& Lewis, 1972). Fleming et al. (1972) demonstrated that, in at least some cases, resistance was conferred by a transferable plasmid ( $\mathrm{R}$ factor). We have tested trimethoprim-resistant strains of Enterobacteriaceae isolated in hospitals in the U.K. The levels of resistance varied considerably. High-level resistance (ability to grow on medium containing $1000 \mu \mathrm{g}$ or more trimethoprim/ml) was always $\mathrm{R}$ factor determined. The determining plasmids all belonged to compatibility group W (Hedges \& Datta, I97I).

The $\mathrm{R}^{+}$strains constituted a high proportion of trimethoprim-resistant cultures from three of seven hospitals. A majority of them were Klebsiella spp. with uniform and unusual biochemical characteristics. Cultures from the other four hospitals were resistant at lower levels and failed to transfer resistance. Most of these were klebsiellae with the biochemical characteristics typical of Klebsiella aerogenes.

\section{METHODS}

Trimethoprim-resistant cultures. Trimethoprim resistance was recognized in hospital diagnostic laboratories, usually by the use of discs containing $\mathrm{I} \cdot 25 \mu \mathrm{g}$ trimethoprim placed on cultures on suitable plates (Waterworth, 1969). Their characteristics are listed in Table I. Each was isolated from a different patient.

Escherichia coli $\mathrm{KI} 2$ strains. $\mathrm{J} 53$ met pro (Clowes \& Hayes, I968); J62 pro his trp (Clowes \& Hayes, I968); $\mathrm{J} 62-\mathrm{I}$ pro his trp nal-r nalidixic acid-resistant mutant of $\mathrm{J} 62 ; \mathrm{J} 62-2$ pro his trp rif-r rifampicin-resistant mutant of $\mathrm{J} 62$; HfrC met (Clowes \& Hayes, 1968).

Plasmids. R factors representative of the known compatability groups are listed in Table 2.

Phage. MS2 (Davis, Strauss \& Sinsheimer, I96I).

Media. Minimal salts agar (Clowes \& Hayes, I968); nutrient broth no. 2 (Oxoid); MacConkey agar (Oxoid $\mathrm{CM}_{7}$ b); diagnostic sensitivity test (DST) agar (Oxoid CM26I). 
Identification. Cultures were tested for production of indole and urease and ability to grow with citrate as carbon source. Strains which were indole + ve, urease - ve, citrate - ve and whose colonial morphology on MacConkey agar was typical of Escherichia coli were recorded as E. coli. All other strains were tested for motility; production of gas from glucose; fermentation of lactose, mannitol, dulcitol, sucrose, salicin and malonate; gelatine liquefaction; MR and VP (Barritt's method) reactions; and decarboxylation of arginine, ornithine and lysine. Methods were as described by Cowan \& Steel (1965). For MR and VP tests cultures were incubated for 2 days at $37^{\circ} \mathrm{C}$. Tables from Cowan \& Steel (1965) were used in naming species.

Determination of minimum inhibitory concentration (MIC). DST agar plates containing $4 \%$ lysed horse blood and serial concentrations of trimethoprim were seeded with suspensions of bacteria at a dilution to give separate colonies on control plates. Plates were incubated overnight at $37^{\circ} \mathrm{C}$ and the MIC recorded as that which prevented visible growth.

Transfer of $R$ factors from wild strains to Escherichia coli KI2. Overnight broth cultures of the wild strain and a KI2 derivative (J62-I if the wild strain was nal-s, $\mathrm{J}_{22-2}$ if the wild strain was nal-r) were mixed in a ratio of one part donor to nine parts recipient and incubated overnight at $37^{\circ} \mathrm{C}$. The mixture was plated on minimal salts agar with appropriate amino acids, nalidixic acid, $25 \mu \mathrm{g} / \mathrm{ml}$ (or rifampicin, $50 \mu \mathrm{g} / \mathrm{ml}$ ) and trimethoprim, $5 \mu \mathrm{g} / \mathrm{ml}$, and the plates were incubated for $24 \mathrm{~h}$ at $37^{\circ} \mathrm{C}$. Colonies appearing on these plates were streaked on DST plates containing $4 \%$ lysed horse blood and trimethoprim, $5 \mu \mathrm{g} / \mathrm{ml}$. Well isolated colonies with appearance typical of $E$. coli $\mathrm{KI} 2$ were identified by their nutritional requirement and their sensitivity pattern was tested. Clones satisfying all tests were taken to be $\mathrm{R}^{+}$ derivatives of $\mathrm{J} 62-\mathrm{I}$ (or $\mathrm{J} 62-2$ ).

Transfer of $R$ factors between lines of Escherichia coli KI2. As described in Coetzee, Datta \& Hedges (I972).

Transfer of the $R$ factor S-a to Klebsiella strains. When J53 (S-a) was used as donor to Klebsiella strains the procedure was that used for matings between strains of Escherichia coli KI2 except that mating mixtures were incubated overnight and plated on minimal medium with sodium citrate $(0.2 \%, \mathrm{w} / \mathrm{v})$ as carbon source and $7 \mu \mathrm{g}$ kanamycin $/ \mathrm{ml}$ of medium.

Determination of $f i$ character. As described in Datta et al. (I97I).

Tests for compatability. As described in Coetzee et al. (1972).

\section{RESULTS}

Minimal inhibitory concentrations of trimethoprim. The MICs are listed in Table I.

Transfer of trimethoprim resistance. Trimethoprim ( $\mathrm{Tp}$ ) resistance was transferred to Escherichia coli KI 2 (J62-I or J62-2) from I9 strains, always accompanied by sulphonamide (Su) resistance. In many cases resistance to other drugs was also transferred, although selection was for $T p$ resistance only (Table I).

Transfer between lines of Escherichia coli $\mathrm{K} \mathrm{I} 2$. The Tp-resistant $\mathrm{J62-2}$ strains were used as donors in matings with $\mathrm{J} 53$ and $\mathrm{HfrC}$, selection being for Tp-resistance. In 13 cases Su- and Tp-resistance was transferred without resistance to other drugs. The HfrC R + derivatives of these $\mathrm{r}_{3}$ were visibly lysed by phage MS2 (i.e. the R factors were $f^{-}$). These were $\mathrm{R}_{3} 88, \mathrm{R}_{405}$, R406, R407, R408, R4II, R4I3, R419, R420, R42I, R422, R423, R424. In the other six cases, resistance to antibiotics (one or more of the following: ampicillin, streptomycin, tetracycline, chloramphenicol) was always transferred with resistance to $\mathrm{Su}$ and $\mathrm{Tp}$. None of the HfrC R+ derivatives of these crosses were visibly lysed by MS2 (i.e. $f+\mathrm{R}$ factors were 
Table I. Naturally occurring trimethoprim-resistant bacteria

\begin{tabular}{|c|c|c|}
\hline No. & Source* & Species \\
\hline D769§ & $\mathbf{P}$ & Escherichia coli \\
\hline D798 & $\mathrm{P}$ & E. coli \\
\hline D80o & $\mathbf{P}$ & E. coli \\
\hline D795 & $\mathbf{P}$ & Klebsiella aerogenes \\
\hline D770॥ & $\mathrm{P}$ & Klebsiella $\mathrm{sp}$ \\
\hline D792 & $\mathbf{P}$ & Klebsiella $\mathrm{sp}$ \\
\hline D793 & $\mathbf{P}$ & Klebsiella $\mathrm{sp}$ \\
\hline D794 & $\mathbf{P}$ & Klebsiella $\mathrm{sp}$ \\
\hline D796 & $\mathbf{P}$ & Klebsiella sp \\
\hline D797 & $\mathbf{P}$ & Klebsiella sp \\
\hline D799 & $\mathrm{P}$ & Klebsiella sp \\
\hline D80I & $\mathrm{U}$ & Klebsiella $\mathrm{sp}$ \\
\hline D802 & $\mathrm{U}$ & Klebsiella $\mathrm{sp}$ \\
\hline D805 & $\mathrm{U}$ & Klebsiella $\mathrm{sp}$ \\
\hline D806 & $\mathrm{U}$ & Klebsiella sp \\
\hline D808 & W & Klebsiella sp \\
\hline D809 & W & Klebsiella $\mathrm{sp}$ \\
\hline D810 & W & Klebsiella sp \\
\hline $\mathrm{D} 8 \mathrm{II}$ & W & Klebsiella sp \\
\hline D829 & $\mathrm{U}$ & Klebsiella sp \\
\hline $\mathrm{D} 830$ & $\mathrm{U}$ & Klebsiella sp \\
\hline D83 I & $\mathbf{P}$ & E. coli \\
\hline D832 & $\mathbf{P}$ & E. coli \\
\hline D833 & $\mathbf{P}$ & E. coli \\
\hline D834 & $\mathbf{U}$ & E. coli \\
\hline D837 & $\mathbf{P}$ & E. coli \\
\hline D845 & $\mathrm{H}$ & E. coli \\
\hline 0852 & B & E. coli \\
\hline D855 & B & E. coli \\
\hline D865 & G & E. coli \\
\hline D847 & $\mathbf{M}$ & $K$. pneumoniae \\
\hline D835 & $\mathrm{U}$ & $K$. aerogenes \\
\hline $\mathrm{D} 836$ & $\mathrm{U}$ & $K$. aerogenes \\
\hline D807 & $\mathrm{U}$ & $K$. aerogenes \\
\hline $\mathrm{D} 838$ & $\mathrm{H}$ & K. aerogenes \\
\hline D839 & $\mathrm{H}$ & $K$. aerogenes \\
\hline D840 & $\mathrm{H}$ & $K$. aerogenes \\
\hline D84I & $\mathrm{H}$ & $K$. aerogenes \\
\hline $\mathrm{D} 842$ & $\mathbf{H}$ & $K$. aerogenes \\
\hline D843 & $\mathrm{H}$ & $K$. aerogenes \\
\hline D844 & $\mathrm{H}$ & K. aerogenes \\
\hline D846 & $\mathrm{H}$ & $K$. aerogenes \\
\hline D848 & $\mathbf{M}$ & $K$. aerogenes \\
\hline D849 & $\mathbf{M}$ & $K$. aerogenes \\
\hline D850 & B & $K$. aerogenes \\
\hline $\mathrm{D} 85 \mathrm{I}$ & B & K. aerogenes \\
\hline D853 & B & $K$. aerogenes \\
\hline D854 & B & $K$. aerogenes \\
\hline $\mathrm{D} 86 \mathrm{I}$ & G & K. aerogenes \\
\hline $\mathrm{D} 862$ & G & $K$. aerogenes \\
\hline $\mathrm{D} 864$ & $\mathbf{G}$ & K. aerogenes \\
\hline
\end{tabular}

$\mathrm{MIC}$
$(\mu \mathrm{g} / \mathrm{ml})$

Resistance transferred to J62-I or $\mathrm{J} 62-2 \ddagger$ (selection $\mathrm{R}$ factor pattern $\uparrow$

SuTp

TSuTp

SuTp

ATCSuTpNal

ASTCSuTp

ASTCSuTp

ASTCSuTpNal

ASTCSuTp

ASTCSuTp

ASCSuTp

ASTCSuTpNal

ASTCSuTp

ASTCSuTpNal

ASCSuTpFuNal

ASTCSuTp

ASTCSuTp

ASTCSuTpFuNal

ASTCSuTp

ASTCSuTp

ASCSuTp

ASSuTp

SuTp

SuTp

SuTp

STSuTp

ASSuTp

ASTKSuTpFuNal

ASKSuTpNal

ASKSuTp

ASTCSuTp

ASTCKSuTpFuNal

ASSuTp

ACSuNal

ASTSuTpFu

ASCSuTpFuNal

ASTCKSuTpFu

ASTCSuTpFuNal

ASTCKSuTp

ATpNal

ASTCKSuTpNal

ASTCKSuTpNal

ASTCKSuTpFuNal

ASTCSuTp

ASTCKSuTpFuNal

ACTpFuNal

ASSuTp

ASCSuTpFuNal

ACTpFuNal

ASCKSuTpNal

ASTCKSuTpNal

ASTCSuTpNal for $\mathrm{Tp}$ resistance) no.

SuTp

TSuTp

SuTp

SuTp

STCSuTp

STCSuTp

ASTCSuTp

ASTCSuTp

SuTp

SuTp

STCSuTp

ASTCSuTp

ASTCSuTp

SuTp

ASTCSuTp

SuTp

ASTCSuTp

ASTCSuTp

ASTCSuTp

$\mathrm{R}_{388}$

R409

R4I I

R406

R389

$\mathrm{R} 403$

R404

R405

R407

R408

R4IO

R4I2

R4I3

R4I9

$>1000$

$>1000$

$>1000$

$>1000$

$>1000$

$>1000$

64

32

256

64

I 28

I6

32

32

32

I 28

I 28

32

328
128

64

64

256

I 28

I 28

256

I 28

I 28

32

I 28

I 6

8

32

256

I 28

256

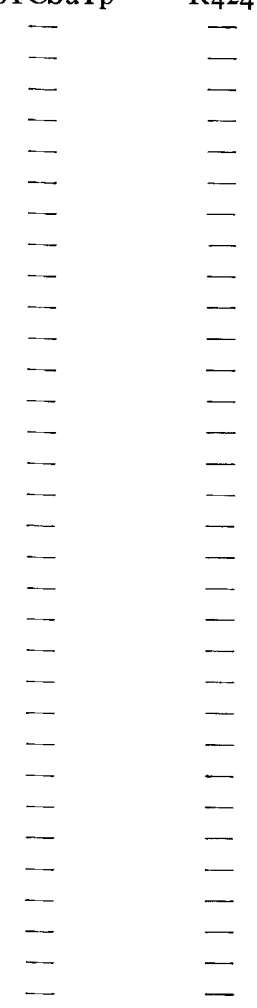

* P, St Pancras Branch, University College Hospital, London; U, University College Hospital, Gower St, London; W, Whittington Hospital, Highgate, London; H, Hammersmith Hospital, London; M, West Middlesex Hospital, London; B, Royal Infirmary, Bristol; G, Royal Infirmary, Glasgow.

$\dagger$ Symbols for resistance to: ampicillin (A), streptomycin (S), tetracycline (T), chloramphenicol (C), kanamycin (K), sulphonamides (Su), trimethoprim (Tp), furazolidone (Fu), nalidixic acid (Nal).

$\$$ Single clone, selected on minimal salts agar supplemented with proline, histidine, tryptophan, trimethoprim and either nalidixic acid or rifampicin.

$\S$ D769 was strain ECI (Fleming et al. I972). It was distinguishable from D798 and D800 (see text).

If D770 was strain KAI (Fleming et al. 1972).

if Strains listed as Klebsiella sp. were MR+ VP+ malonate-ve (see text). 
present). $\mathrm{R}_{3} 89$ was further studied as an example of these. (R389 was the $\mathrm{R}$ factor reported as $f^{+}$by Fleming et al. I972.)

When $\mathrm{J} 62-\mathrm{I}\left(\mathrm{R}_{3} 89\right)$ was used as donor, and selection was made for $\mathrm{Tp}$ resistance, every clone tested (50/50) was resistant to streptomycin (S), tetracycline (T) and chloramphenicol (C), as well as to Su and Tp even though mating was interrupted after $5 \mathrm{~min}$. When chloramphenicol was used for selection, resistant recipients had either of two resistance patterns, STCSu or STCSuTp. HfrC with either pattern showed no visible lysis by phage MS2, thus the $\mathrm{fi}^{+}$character was always associated with resistance to STCSu.

Compatibility of Tp-resistance plasmids. The first $\mathrm{R}$ factor conferring $\mathrm{Tp}$-resistance to be identified, $\mathrm{R}_{3} 88$, was transferred from $\mathrm{J}_{62}-\mathrm{I}$ to $\mathrm{J} 53$ and $\mathrm{J} 53$ carrying plasmids of diverse compatibility groups. No exclusion of $\mathrm{R} 388$ by any resident plasmid was observed. However, $\mathrm{R}$ factor S-a, a W plasmid, was eliminated from all $\mathrm{R}_{3} 88$ + clones tested (20/20). $\mathrm{R}_{3} 88$ coexisted stably with all the other $\mathrm{R}$ factors. The frequency of transfer of $\mathrm{R} 388$ was reduced by the presence of $\mathrm{RP}_{4}$ when both factors were present in the donor culture (Table 2).

$\mathrm{R}$ factor S-a was introduced into lines of J62-I or J62-2 carrying each of the other transmissible Tp-resistance plasmids R389, R403, R404, R405, R406, R407, R408, R409, R4I0, R4II, R4I2, R4I3, R4I9, R420, R42I, R422, R423 and R424. In no case did the resident plasmid exclude transfer of S-a but in every case resistance to trimethoprim was lost. With $\mathrm{R}_{389}$ resistance to tetracycline was retained (the continued presence of the streptomycin, chloramphenicol and sulphonamide resistances of $\mathrm{R}_{3} 89$ could not be detected in the presence of S-a). S-a was introduced into naturally occurring Klebsiella strain D829 (Table I) which was highly resistant to $\mathrm{Tp}$ (but did not transfer resistance) and $\mathrm{Tp}$ resistance was eliminated. We were unable to detect transfer of S-a to D830.

Distribution of $R$ factors determining Tp-resistance. Of the 20 wild strains in which $\mathrm{Tp}$ resistance was determined by a W plasmid, I 7 were Klebsiella spp. and three were Escherichia coli. Of the 17 Klebsiella strains one, D795, was typical Klebsiella aerogenes (Cowan \& Steele, I965) the other I6 were all MR + VP + malonate - ve i.e. could not be classified as either $K$. aerogenes or $K$. pneumoniae. D830 was similar and was resistant to $>1000 \mu \mathrm{g}$ trimethoprim $/ \mathrm{ml}$, but we have no direct evidence that its Tp resistance was plasmid-determined. Of the $3 E$. coli, one (D769) belonged to O group 18 (Fleming et al. 1972): the other two could not be grouped with a limited range of antisera, but were not Or 8 , and differed from one another biochemically. D798 fermented dulcitol, D80o did not (Table I). The one $K$. aerogenes and the three $E$. coli all came from one hospital (University College Hospital, St. Pancras Branch). All the strains carrying Tp resistance plasmids were isolated from patients at University College Hospital (Gower St. and St Pancras branches, which are over a mile apart) and the Whittington Hospital, Highgate.

Strains of bacteria resistant to trimethoprim but with MICs $<1000 \mu \mathrm{g} / \mathrm{ml}$ are listed in Table I. There were nine isolates of Escherichia coli, 20 of typical Klebsiella aerogenes, and one of $K$. pneumoniae. None transferred $\mathrm{Tp}$ resistance. They were isolated at University College Hospital, Hammersmith Hospital, the West Middlesex Hospital, Bristol Royal Infirmary and the Royal Infirmary, Glasgow.

\section{DISCUSSION}

In all naturally occurring strains of bacteria resistant to $1000 \mu \mathrm{g}$ trimethoprim $/ \mathrm{ml}$ medium, the resistance was determined by a plasmid of the $\mathrm{W}$ compatibility group. Only with $\mathrm{D} 830$ have we no direct evidence for this statement. Its $\mathrm{Tp}$ resistance was non-transmissible and the strain failed to accept $\mathrm{S}-\mathrm{a}$, so elimination by a W plasmid could not be tested. 


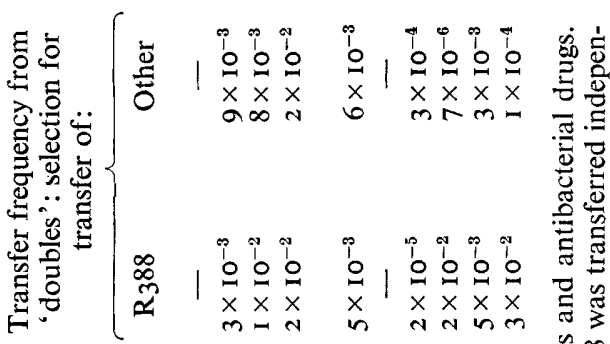

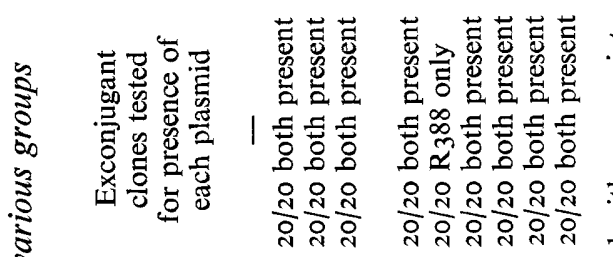

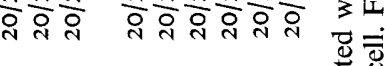

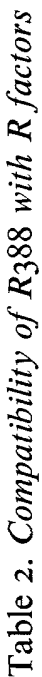

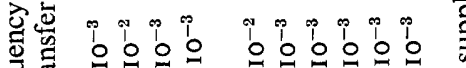


The only previously identified $\mathrm{R}$ factors of the $\mathrm{W}$ group did not confer resistance to trimethoprim. They were derived from strains of Shigella and Aeromonas from Japan (Hedges \& Datta, 197I) and of Proteus rettgeri from Greece (Kontomichalou, I97I; Coetzee et al. 1972). Like the ones described here they showed mutual incompatibility but no mutual surface exclusion. Most of the Tp-resistance plasmids were self-transmissible and conferred resistance to Su and Tp only. One Tp plasmid (in D829) was not transmissible but was shown to be a $\mathrm{W}$ plasmid by elimination by $\mathrm{R}$ factor $\mathrm{S}-\mathrm{a}$. We think it likely that the Tp resistance of D830 was of the same nature. In some cases, e.g. D770, resistance to Tp and Su was transferred only in company with resistance to other drugs. From the KI 2 recipients, Tp resistance could be eliminated by introduction of S-a. We believe the original bacteria were 'doubles', carrying non-self-transmissible W plasmids conferring resistance to Tp and $\mathrm{Su}$ and $f i+\mathrm{R}$ factors, bearing the other resistance markers. Transmissibility was the only difference observed among the Tp plasmids which suggests that they were all produced by replication of a single original replicon. This was also suggested by their geographical distribution which was confined to three London hospitals, separated from one another by several miles. Between two of them (the two branches of University College Hospital) there is some interchange of patients and staff but with the third (the Whittington) exchange is limited to annual rotation of some junior medical staff.

The hosts for the $\mathrm{R}$ factors were Enterobacteriaceae, isolated from infected patients. Most of them were members of the genus Klebsiella with unusual biochemical characteristics, not conforming with the definitions of any Klebsiella species (Cowan \& Steele, I965). All but one of the R + Klebsiella strains were closely similar biochemically, although their resistance patterns varied, and it seems likely that they were members of a single clone present in these hospitals in the Camden area of London. The $\mathrm{R}$ factors had been transferred to one strain of typical Klebsiella aerogenes and to three distinguishable strains of Escherichia coli. The spread of the predominant host and the spread of the $\mathrm{R}$ factor to other bacteria demonstrates the two mechanisms by which an $\mathrm{R}$ factor may be distributed in nature. There is a parallel between the dissemination of trimethoprim resistance, determined by $\mathrm{W}$ plasmids, and that of carbenicillin resistance, mediated by P plasmids in Pseudomonas aeruginosa and Proteus and Klebsiella spp. in the MRC Industrial Injuries and Burns Unit in Birmingham (Datta et al. I97I; Roe \& Lowbury, 1972). In each case the R factor belonged to a compatibility group seldom or never described before; thus apparently previously rare types of plasmid are spreading epidemically. The probable selective advantage enjoyed by the $P$ plasmids in the Burns Unit was their ability to infect $P$. aeruginosa, conferring resistance to carbenicillin. For the $\mathrm{W}$ plasmids it is that they confer resistance to a new drug trimethoprim.

Since trimethoprim is widely used, and not especially in the three hospitals where the $R$ factors were found, we anticipate a dissemination of the $\mathrm{R}$ factor to other hospitals and through bacterial populations generally.

We are very grateful to Dr Joan Stokes, Miss Pamela M. Waterworth, Dr R. N. Grüneberg, Dr Felicity M. Anderson, Dr R. W. Lacey and Dr R. W. A. Girdwood for sending us cultures of trimethoprim-resistant bacteria and to $\mathrm{Mr} \mathrm{R}$. A. Lambert for serotyping Escherichia coli strains. 


\section{REFERENCES}

Clowes, R. C. \& HAYes, W. (1968). Experiments on Microbial Genetics. Oxford \& Edinburgh: Blackwell Scientific Publications.

Coetzee, J. N., Datta, N. \& Hedges, R. W. (1972). R factors from Proteus rettgeri. Journal of General Microbiology 72 (in the press).

CowAN, S. T. \& STEEL, K. J. (I965). Manual for the Identification of Medical Bacteria. Cambridge: University Press.

Datta, N. \& Hedges, R. W. (I97I). Compatibility groups among $f^{-}$R factors. Nature, London 234 , 222-223.

Datta, N., Hedges, R. W., Shaw, E. J., Sykes, R. \& Richmond, M. H. (1971). Properties of an R factor from Pseudomonas aeruginosa. Journal of Bacteriology 108, I 244-I 249.

Davis, J. E., Strauss, J. H. \& Sinsheimer, R. L. (196I). Bacteriophage MS2: another RNA phage. Science, New York 134, 1427.

Fleming, M. P., Datta, N. \& Grüneberg, R. N. (1972). Trimethoprim resistance determined by R factors. British Medical Journal I, 726-728.

Hedges, R. W. \& DatTa, N. (I97I). $f^{-}$R factors conferring chloramphenicol resistance. Nature, London $\mathbf{2 3 4}$, 220-22I.

Hedges, R. W. \& Datta, N. (1972). Ri24, an $f^{+}$R factor of a new compatibility class. Journal of General Microbiology 7I, 403-405.

KontomichaloU, P. (197I). R factors controlling resistance to the penicillins. Habilitation Thesis, University of Athens.

Lacey, R. W., Gillespie, W. A., Bruten, D. M. \& Lewis, E. (1972). Trimethoprim resistant coliforms. Lancet $\mathrm{i}, 409-410$.

Lawn, A. M., Meynell, E., Meynell, G. G. \& Datta, N. (1967). Sex pili and the classification of sex factors in the Enterobacteriaceae. Nature, London 216, 343-346.

LEBEK, G. \& WIEDMER, E. (I97I). Empfindlichkeit menschlicher Krankheitserreger gegen das KombinationsTherapeutikum Sulfamethoxazol-Trimethoprim in vitro. Schweizerische Medizinische Wochenschrift ror, 1385-1390.

Roe, E. \& Lowbury, E. J. L. (1972). Changes in antibiotic sensitivity patterns of Gram-negative bacilli in burns. Journal of Clinical Pathology 25, 176-178.

Waterworth, P. M. (1969). Practical aspects of testing sensitivity to trimethoprim and sulphonamide. Postgraduate Medical Journal (Suppl) 45, 2 I-27. 